\title{
1 p36 deletion is a marker for tumour dissemination in microsatellite stable stage II-III colon cancer
}

\author{
Markus Mayrhofer ${ }^{1}$, Hanna Göransson Kultima', Helgi Birgisson², Magnus Sundström³, Lucy Mathot ${ }^{3}$, \\ Karolina Edlund ${ }^{3,5}$, Björn Viklund ${ }^{1}$, Tobias Sjöblom³, Johan Botling ${ }^{3}$, Patrick Micke ${ }^{3}$, Lars Påhlman², \\ Bengt Glimelius ${ }^{4}$ and Anders Isaksson ${ }^{1^{*}}$
}

\begin{abstract}
Background: The clinical behaviour of colon cancer is heterogeneous. Five-year overall survival is $50-65 \%$ with all stages included. Recurring somatic chromosomal alterations have been identified and some have shown potential as markers for dissemination of the tumour, which is responsible for most colon cancer deaths. We investigated 115 selected stage II-IV primary colon cancers for associations between chromosomal alterations and tumour dissemination.

Methods: Follow-up was at least 5 years for stage II-III patients without distant recurrence. Affymetrix SNP 6.0 microarrays and allele-specific copy number analysis were used to identify chromosomal alterations. Fisher's exact test was used to associate alterations with tumour dissemination, detected at diagnosis (stage IV) or later as recurrent disease (stage II-III).
\end{abstract}

Results: Loss of 1p36.11-21 was associated with tumour dissemination in microsatellite stable tumours of stage II-IV (odds ratio $=5.5$ ). It was enriched to a similar extent in tumours with distant recurrence within stage II and stage III subgroups, and may therefore be used as a prognostic marker at diagnosis. Loss of 1p36.11-21 relative to average copy number of the genome showed similar prognostic value compared to absolute loss of copies. Therefore, the use of relative loss as a prognostic marker would benefit more patients by applying also to hyperploid cancer genomes. The association with tumour dissemination was supported by independent data from the The Cancer Genome Atlas.

Conclusion: Deletions on 1p36 may be used to guide adjuvant treatment decisions in microsatellite stable colon cancer of stages II and III.

Keywords: Colon cancer, Prognostic marker, Allele-specific copy number analysis, Genome duplication, 1p36, Metastasis, Tumour dissemination

\section{Background}

Colon cancer is a heterogeneous disease in terms of clinical behaviour with an overall 5-year survival of 50-65\%. Except for postoperative mortality all colon cancer-related deaths are caused by dissemination of the tumour (metastatic disease), present in $20-25 \%$ of patients at the time of diagnosis, and appearing to a

\footnotetext{
* Correspondence: anders.isaksson@medsci.uu.se

'Science for Life Laboratory, Department of Medical Sciences, Uppsala University, Box 3056, Uppsala 750 03, Sweden

Full list of author information is available at the end of the article
}

similar extent during follow-up in individuals who were found to be metastasis-free at diagnosis. After surgical resection of the primary tumour, adjuvant chemotherapy may reduce the risk of subsequent relapse by eradicating subclinical tumour deposits. Prognostic markers are warranted in patient subgroups where they could influence the choice of treatment, such as selecting adjuvant therapy in stage II-III patients. TNM staging has relatively low predictive value, but is currently the only validated prognostic tool. Improved molecular prognostic markers could have a potential to reduce both over- and 
under-treatment by identifying patients with the greatest potential benefit from adjuvant therapy.

The mutational landscape of colon cancer has been explored in detail [1]. Colon cancers with microsatellite instability (MSI) have few or no somatic copy number alterations (CNAs). Microsatellite stable (MSS) colon cancers frequently have mutations in tumour suppressor genes such as APC and TP53. MSS colon cancers also frequently have chromosomal instability (CIN) which results in numerous CNAs. Multiple molecular prognostic markers such as MSI (excluding low-level MSI [2,3]), loss of $18 \mathrm{q}$ and reduced SMAD4 expression have been suggested [4-7]. Other CNAs that have been associated with survival or tumour dissemination include loss of $1 \mathrm{p}$, $4 p, 8 p, 9 q, 10 p 15 q, 19 p$ and $20 p$ and gain of $8 q$ and $20 q$ [1,8-10]. Unfortunately, findings vary considerably between studies and there is no consensus set of CNAs associated with tumour dissemination, i.e. prognosis for patients without metastasis at diagnosis.

Copy number analysis of tumour tissue is complicated by unknown ploidy of the tumour cells, by normal cells in the tumour tissue, and by subclonal CNAs. Bioinformatic tools such as TAPS [11] use bi-allelic probe signals from SNP arrays to estimate absolute allele-specific copy numbers in tumour cells. Allele-specific copy number analysis has been used to estimate frequency of hyperploidy and whole-genome duplication in multiple cancer types [12].

This study aimed to identify CNAs in colon cancer that may be used at diagnosis to predict risk for tumour dissemination in stage II-III patients. DNA from resected stage II-IV colon cancer primary tumours were analysed on Affymetrix SNP 6.0 arrays. Bioinformatic analysis identified deletion on 1p36 as a marker for tumour dissemination.

\section{Methods}

\section{Study population}

The study cohort included 116 patients operated for colorectal cancer between 1985 and 2006 at the Uppsala University hospital and at Västerås district general hospital between 2000-2003, with fresh frozen tissue samples available. We aimed at selecting between 20-25 cases each with stages II and III with and without distant recurrence and stage IV. Morphological and clinical parameters were retrieved from the original pathology reports. Patients with a history of preoperative therapy or with a surgical or pathology report suggesting a non-radical resection margin (R1 or $\mathrm{R} 2$ resection) were excluded. To secure the quality of disease staging, patients with stage II disease were only included if at least 10 lymph nodes were analysed. Patients with disease stage II-III and no recurrence were only included if the follow-up time was longer than 5 years. Tumour cell content was required to be at least $40 \%$ in the frozen tissue block. The study design was chosen to have few factors confounding an association between the tumour genome at diagnosis (surgery) and development of distant metastasis. Clinical and histological characteristics are presented in Table 1. Adjuvant chemotherapy, chiefly with a fluoropyrimidine alone was given to 22 out of 53 stage II-III patients without recurrence and to 29 out of 40 patients in stages II-III who developed distant metastasis.

\section{DNA extraction}

Genomic DNA was extracted from $10 \mu \mathrm{m}$ sections of the fresh frozen tissue using QIAamp DNA mini kit (QIAGEN GmbH, Hilden, Germany) according to the

Table 1 Clinical and histopathological data

\begin{tabular}{|c|c|c|c|c|}
\hline & Total & $\begin{array}{c}\text { Stage II-III } \\
\text { No recurrence }\end{array}$ & $\begin{array}{c}\text { Stage II-IV } \\
\text { Disseminated }\end{array}$ & $p$ \\
\hline \multicolumn{5}{|l|}{ Gender } \\
\hline Male & 48 & 24 & 24 & 0.434 \\
\hline Female & 68 & 29 & 39 & \\
\hline \multicolumn{5}{|l|}{ Location } \\
\hline Right colon & 70 & 33 & 37 & 0.698 \\
\hline Left colon & 46 & 20 & 26 & \\
\hline \multicolumn{5}{|l|}{ Differentiation } \\
\hline Well- Moderately & 89 & 41 & 48 & 0.882 \\
\hline Poor & 27 & 12 & 15 & \\
\hline \multicolumn{5}{|l|}{ Stage } \\
\hline$\|$ & 40 & 25 & 15 & \\
\hline III & 53 & 28 & 25 & \\
\hline IV & 23 & - & 23 & \\
\hline \multicolumn{5}{|l|}{ Tumour size } \\
\hline$<5 \mathrm{~cm}$ & 37 & 12 & 25 & 0.058 \\
\hline$\geq 5 \mathrm{~cm}$ & 78 & 40 & 38 & \\
\hline Unknown & 1 & 1 & & \\
\hline \multicolumn{5}{|l|}{ Mucinous } \\
\hline Yes & 19 & 11 & 8 & 0.243 \\
\hline No & 97 & 42 & 55 & \\
\hline \multicolumn{5}{|l|}{ Perineural invasion } \\
\hline Yes & 3 & 0 & 3 & $0.157^{*}$ \\
\hline No & 113 & 53 & 60 & \\
\hline \multicolumn{5}{|l|}{ Vascular invasion } \\
\hline Yes & 16 & 6 & 10 & 0.479 \\
\hline No & 100 & 47 & 53 & \\
\hline \multicolumn{5}{|c|}{ Microsatellite instability } \\
\hline MSI-High & 24 & 17 & 7 & 0.008 \\
\hline MSS or MSI-Low & 92 & 35 & 57 & \\
\hline
\end{tabular}

*Fisher's exact test, otherwise $x^{2}$. 
manufacturer's recommendations for DNA purification from tissue. Alternatively, genomic DNA was extracted from approximately $25 \mathrm{mg}$ of each fresh frozen colon tissue sample on a Tecan Evo MCA 150 robotic platform using the extraction method described in Mathot et al [13]. DNA concentration was determined using NanoDrop (Thermo Scientific, Wilmington, DE).

\section{MSI analysis}

MSI status was determined using MSI Analysis System, version 1.2 (Promega, Madison, WI) with 6 ng genomic DNA and analysis of five mononucleotide repeat markers (BAT25, BAT26, NR-21, NR-24 and MONO-27). Analyses were performed on a 3130xl genetic analyser (Applied Biosystems, Foster city, CA). According to guidelines from a National Cancer Institute workshop in 1997, samples were denoted MSI-High (MSI-H) if two or more of the five markers show instability, MSI-Low (MSI-L) if only one marker shows instability and microsatellite stable (MSS) if no markers display instability. Recent studies indicate no significant difference between MSI-L and MSS [14] and they were therefore grouped together as MSS in this study.

\section{Microarray analysis}

Array experiments were performed according to standard protocols for Affymetrix GeneChip Mapping SNP 6.0 arrays (Affymetrix Cytogenetics Copy Number Assay User Guide (P/N 702607 Rev2.), Affymetrix Inc., Santa Clara, CA). 500 ng total genomic DNA was digested with a restriction enzyme (Nsp, Sty), ligated to an appropriate adapter for the enzyme, and subjected to PCR amplification using a single primer. After digestion with DNase I, PCR products were labelled with a biotinylated nucleotide analogue using terminal deoxynucleotidyl transferase and hybridized to the microarray. Hybridized probes were captured by streptavidin-phycoerythrin conjugates using Fluidics Station 450 and arrays were scanned using GeneChip ${ }^{\circ}$ Scanner 3000 7G. SNP array data generated in this study have been deposited at GEO with accession number GSE62875. Independent SNP 6.0 data from TCGA colon adenocarcinoma were retrieved from http://cancergenome.nih.gov.

\section{Data analysis and statistics}

Basic normalisation and segmentation of the microarray data were performed using BioDiscovery Nexus Copy Number 6.0 and the SNP Rank Segmentation algorithm based on Circular Binary Segmentation [15] and default settings. Analyses of absolute allele-specific copy numbers, average ploidy and normal cell content were performed using TAPS [11]. Copy number estimates are included in the Additional file 1.
CNA frequencies (gain to $>2$ copies per cell, loss to $<2$ copies per cell, relative gain to $>1.25^{*}$ individual sample average copy number, relative loss to $<0.67^{*}$ individual sample average copy number, homozygous loss, high gain to $>3$ copies above individual sample average copy number, focal gain and loss of $<1 \mathrm{Mb}$ segments, and loss of heterozygosity) and group comparisons were generated using TAPS. Fisher's exact test was used to estimate statistical significance of observations, generating unadjusted p-values and odds ratios for short segments throughout the genome such that none contained a breakpoint in any sample. A p-value of 0.05 was used as an initial cut-off for significance.

Average copy number or ploidy was calculated as the mean total copy number throughout the autosomes. The difference between the number of autosome arms with $2 \mathrm{~m} 0$ (two copies with minor allele copy 0, i.e. $\mathrm{LOH}$ ) or $4 \mathrm{~m} 2$, and $2 \mathrm{~m} 1$ or $4 \mathrm{~m} 1$ was used as a score for evidence of a genome duplication event (using medians of total and minor allele copies throughout each autosome arm, Formula 1).

$$
\text { WGDscore }=\sum 2 m 0+\sum 4 m 2-\sum 2 m 1-\sum 4 m 1
$$

\section{Results}

Copy number analysis was successful for 115 samples, of which 23 were MSI and 92 MSS. Tumour dissemination was defined as either stage IV with distant metastasis at diagnosis, or stage II-III and recurrence with distant metastasis within 5-years of diagnosis. No association was found between tumour dissemination and gender, tumour location, differentiation, tumour size, mucinous appearance, or neural or vascular invasion (Table 1).

DNA from all samples was analysed using Affymetrix SNP 6.0 arrays and absolute allele-specific copy numbers in the cancer cells were estimated using TAPS [11]. All samples with MSI $(n=23)$ were near diploid with relatively few CNAs; median 3 chromosomes affected and median $87 \mathrm{Mb}$ altered. Nearly all samples with MSS had an abundance of CNAs affecting large proportions of the genome; median 17 chromosomes affected and median $940 \mathrm{Mb}$ altered. Hyperploidy, defined as an average copy number above 2.5, was observed in many MSS samples but showed no association with tumour dissemination $(\mathrm{p}=0.33)$. Associations between specific CNAs and tumour dissemination were investigated separately in MSI and MSS due to the different frequencies of alterations. Sample groups with and without dissemination were compared for differences in the frequency of various types of CNAs (see Methods). Fisher's exact test was used to generate p-values for the null hypothesis that the observed difference in CNA frequency is random. Alteration frequencies and frequency differences in MSS samples are shown in Figure 1. 


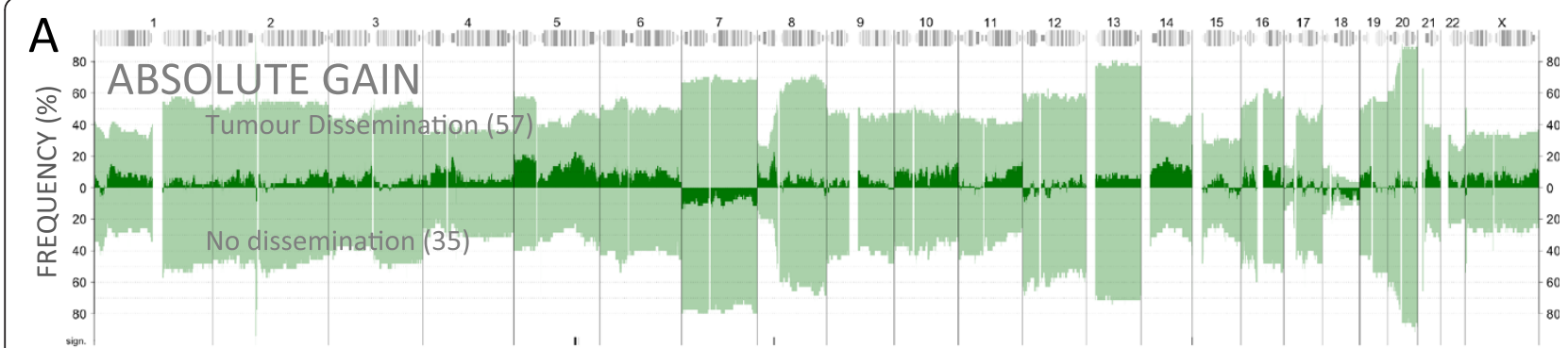

B

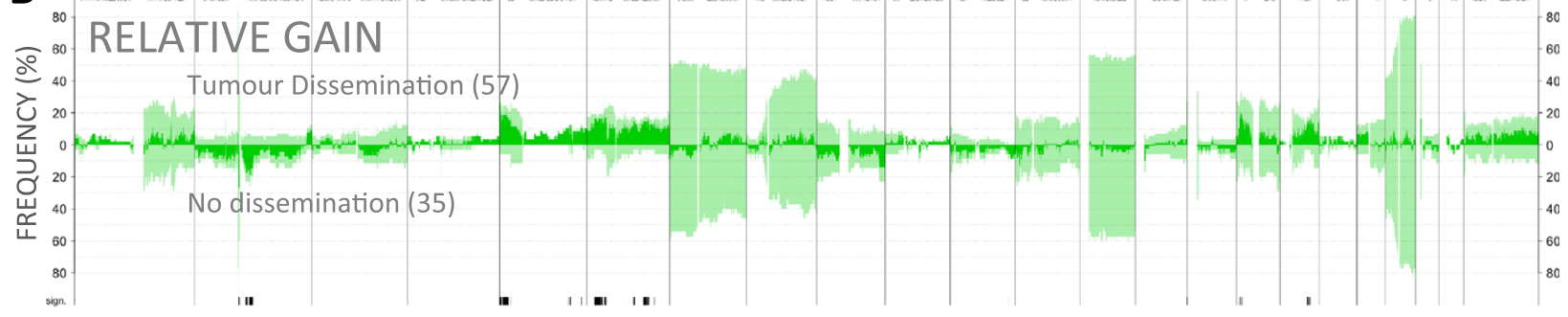

C

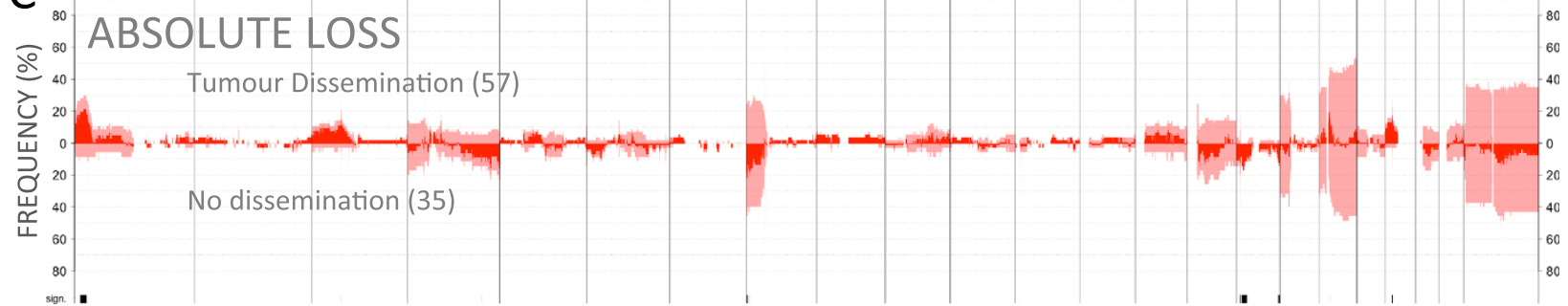

D



E

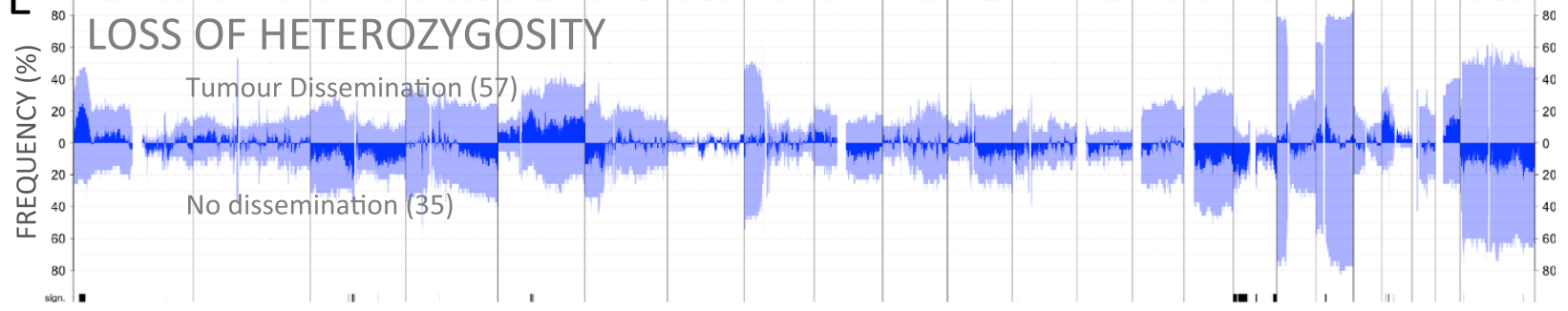

Figure 1 Frequency of copy number alterations. Microsatellite stable tumours with dissemination (stage II-III with distant recurrence, stage IV) and without (stage II-III no recurrence). Frequency difference is shown with a darker colour. Different types of CNAs were analysed separately: A) Gain (to $>2$ copies). B) Relative gain (to $>25 \%$ above individual sample average copy number). C) Loss (to $<2$ copies). D) Relative loss (to $<67 \%$ of individual sample average). E) Loss-of-heterozygosity (no minor allele copy). Regions with significant difference in alteration frequency ( $p<0.05$, Fisher's exact test) are marked by black bars below each panel.

In MSS samples, frequencies of CNAs were similar in samples with and without dissemination, with amplification being more frequent than deletion throughout most chromosomes (Figure 1A, C). Deletion was more frequent than amplification only on chromosomes $8 \mathrm{p}, 17 \mathrm{p}$ and 18. Frequencies of absolute loss (1C) resembled 
those of loss relative to the average copy number of the individual genome (1D) and $\mathrm{LOH}(1 \mathrm{E})$ due to their natural overlap; e.g. absolute loss is also considered $\mathrm{LOH}$. Regions with a significantly different frequency of alteration ( $p<0.05$, Fisher's Exact Test) between the prognostic groups included $1 \mathrm{p} 36$ and $18 \mathrm{q}$ (relative loss), 2q12-14, 5p, 6pq and 17q (relative gain) and 16p (LOH). Additional file 1 includes a complete list of CNA frequency differences (including focal alterations and homozygous loss for which no potential markers were found) and corresponding p-values and odds ratios. In tumours with MSI, some genomic regions including 18q were more commonly altered in disseminated tumours, but the number of MSI samples was limited and statistical significance was not reached for any specific CNA (Additional file 1: Figure S1).

\section{$1 \mathrm{p} 36$ deletion is a marker for tumour dissemination in stages II and III}

We used a publicly available set of colon adenocarcinoma samples from The Cancer Genome Atlas (TCGA) [1] to verify which of our findings could be observed in independent data. The TCGA data set differed from the current study in that progression after diagnosis and MSI status was documented only for subsets of the patients. We observed that large CNAs $(\geq 10 \mathrm{Mb})$ were rare in MSI samples in the current study, affecting less than 5 chromosomes in all but one case, while $>93 \%$ of MSS cases had CNAs on at least 5 chromosomes (Additional file 1: Figure S2A). TCGA samples with known MSI status showed a similar distribution (Additional file 1: Figure S2B). TCGA samples with unknown MSI status and at least 5 chromosomes affected $(n=37)$ were considered CIN and included with known MSS samples in order to maximise the number of samples available for validation. MSS/CIN TCGA cases with metastasis at diagnosis $(n=39)$ were compared with cases without metastasis at diagnosis and with documented survival greater than two years (2-10 years, mean $=5, n=28$ ). Out of all genomic regions with significantly different frequency of alteration and good effect size (odds ratio $\geq 4$ ) in our data, only deletion on 1 p36 (odds ratio $\approx 6$ ) was independently significant in TCGA. We also observed that deletion or LOH on 18q11.2 (short segment including $C A B L E S 1$, odds ratio $\approx 3$ ) was associated with dissemination in both data sets, but with a relatively low effect size (Additional file 1).

Subsets (stage II and stage III separately and together, and with postoperative chemotherapy treated cases removed) of the current study displayed associations (odds ratios) very similar to those of stages II-IV combined, supporting that the deletion may be used as a prognostic marker at diagnosis. Peak odds ratios and independent $95 \%$ confidence intervals are shown in Table 2. Statistics at base-pair resolution throughout the
Table 2 Effect size of 1 p36 loss association with tumour dissemination

\begin{tabular}{lll}
\hline Alteration 1p36.11-21 & Absolute loss & Relative loss \\
\hline OR stage II-IV & $4.5(1.1-25.9)$ & $5.5(1.6-24.5)$ \\
OR stage II & $4.4(0.3-262)$ & $6.5(0.5-368)$ \\
OR stage III & $3.6(0.6-40.3)$ & $3.3(0.7-22.5)$ \\
OR stage II-III & $4.0(0.9-25.1)$ & $4.3(1.13-20.5)$ \\
OR stage II-III no chemotherapy & $8.0(0.9-393)$ & $9.7(1.1-472)$ \\
OR TCGA & $4.1(1.0-25.1)$ & $2.8(0.8-11.6)$ \\
Total frequency & $17 \%$ & $30 \%$ \\
\hline
\end{tabular}

region are included in Additional file 1. Absolute loss (fewer than 2 copies remaining) and relative loss (fewer than genome average number of copies remaining) were similarly associated with tumour dissemination, though relative loss was more frequently observed (Table 2). For relative loss on $1 \mathrm{p} 36$, the difference in frequency between the prognostic groups of the current study (stage II-IV) exceeded $30 \%$ on $1 \mathrm{p} 36.13$ but was similar throughout 1p36.11-21 (Figure 2A). For the TCGA validation set the difference in frequency exceeded $20 \%$ on $1 \mathrm{p} 36.11$ and was similar throughout 1p36.11-13 (2B). Total frequency of relative loss displayed very similar profiles in both data sets and peak frequency of loss could be observed near $27 \mathrm{Mb}(2 \mathrm{C})$. The difference in frequency between the prognostic groups could be confirmed in the TCGA data set (2B), but a single gene or region responsible for the worse prognosis could not be pinpointed.

\section{Duplication of the genome is not associated with prognosis or relative loss on 1 p36}

In both the current study and the TCGA validation data, $41 \%$ of MSS/CIN samples were found to have an average copy number or ploidy above 2.5 . Hyperploid genomes may be the effect of whole genome duplications (WGD), which have been implicated in tumours and observed in cancer cell lines [16,17]. Allele-specific copy number analysis has been used to identify WGD as a frequent event in a variety of cancers including colon cancer, where the frequency of WGD has been estimated to approximately $50 \%$ [12]. We investigated evidence of hyperploidy (average copy number $>2.5$ ) and WGD in the current study and in the TCGA validation set. For chromosomes present in 4 copies, a WGD event is more likely to have produced 2 copies of each homolog than triplication of one homolog, which is the more likely outcome of successive amplification events leading to 4 copies. Similarly where 2 copies are present, $\mathrm{LOH}$ is more likely observed if a WGD event has taken place (after loss of one copy, or followed by loss of two random copies) than if not. We used these assumptions to develop a score sensitive to WGD (see Methods) that 


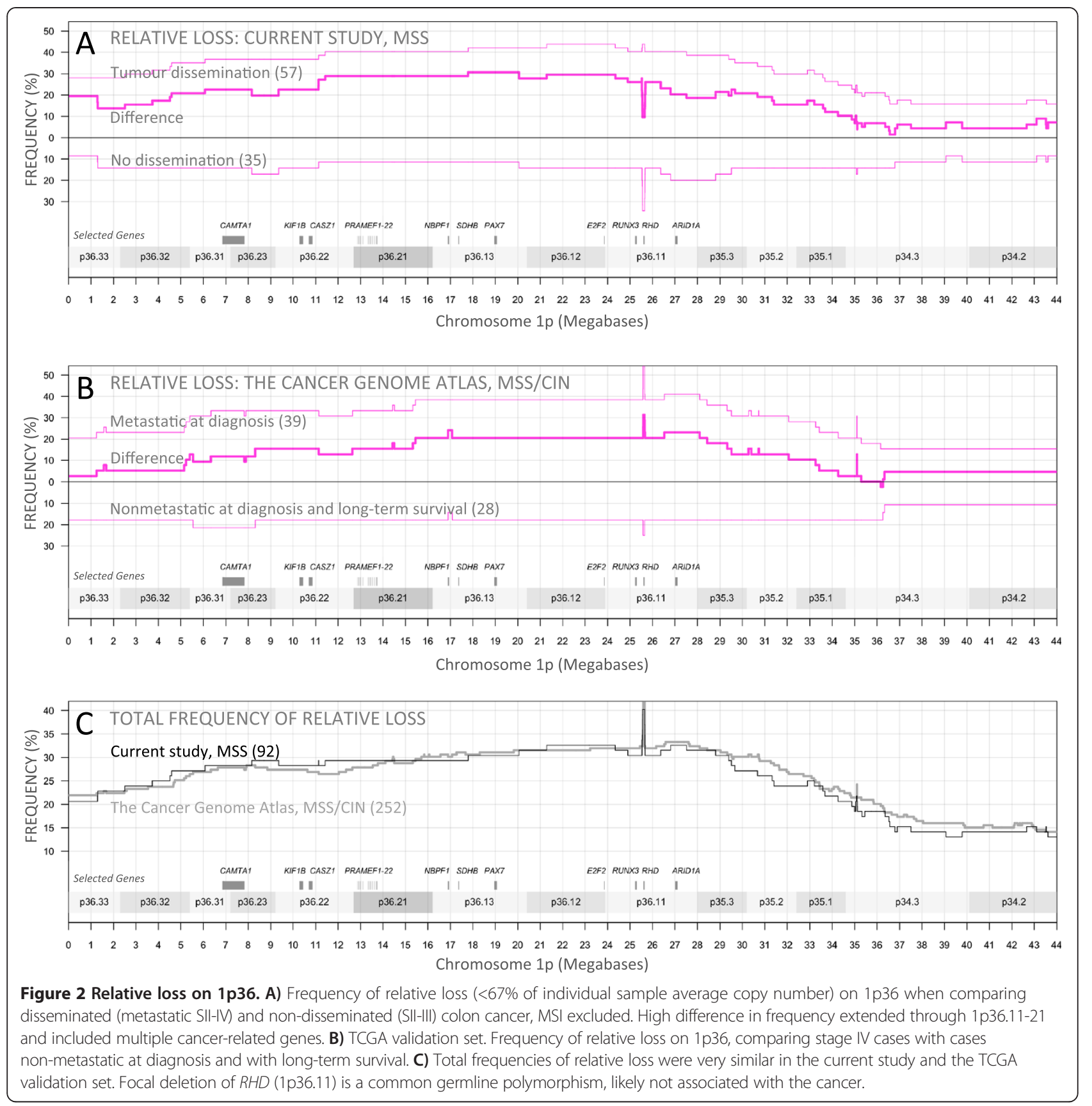

would not be directly influenced by the average copy number of the genome. Not surprisingly, average ploidy correlated strongly with WGD score (Figure 3AB). Bimodal distributions of the WGD score for both the current study and the TCGA validation set suggest two groups of samples; one having undergone WGD and the other not. WGD appeared to have occurred in most genomes with hyperploidy and in about one third of all samples. Average ploidy or WGD score did not associate with prognosis or relative loss on $1 \mathrm{p} 36$ ( $\mathrm{p}>0.2$, current study MSS, logistic regression). However, absolute loss on 1 p36 was strongly associated with, and nearly exclusive to near diploid genomes $\left(\mathrm{p}<10^{-5}\right)$.

\section{Discussion}

Treatment decisions for colon cancer patients are based on TNM staging, where stage III patients most often receive adjuvant chemotherapy while stage II patients (due to the low risk for metastatic relapse) are only treated beyond surgical resection if some risk factors are observed. Molecular markers have the potential to guide the use of adjuvant treatment to minimize over- and under-treatment. 

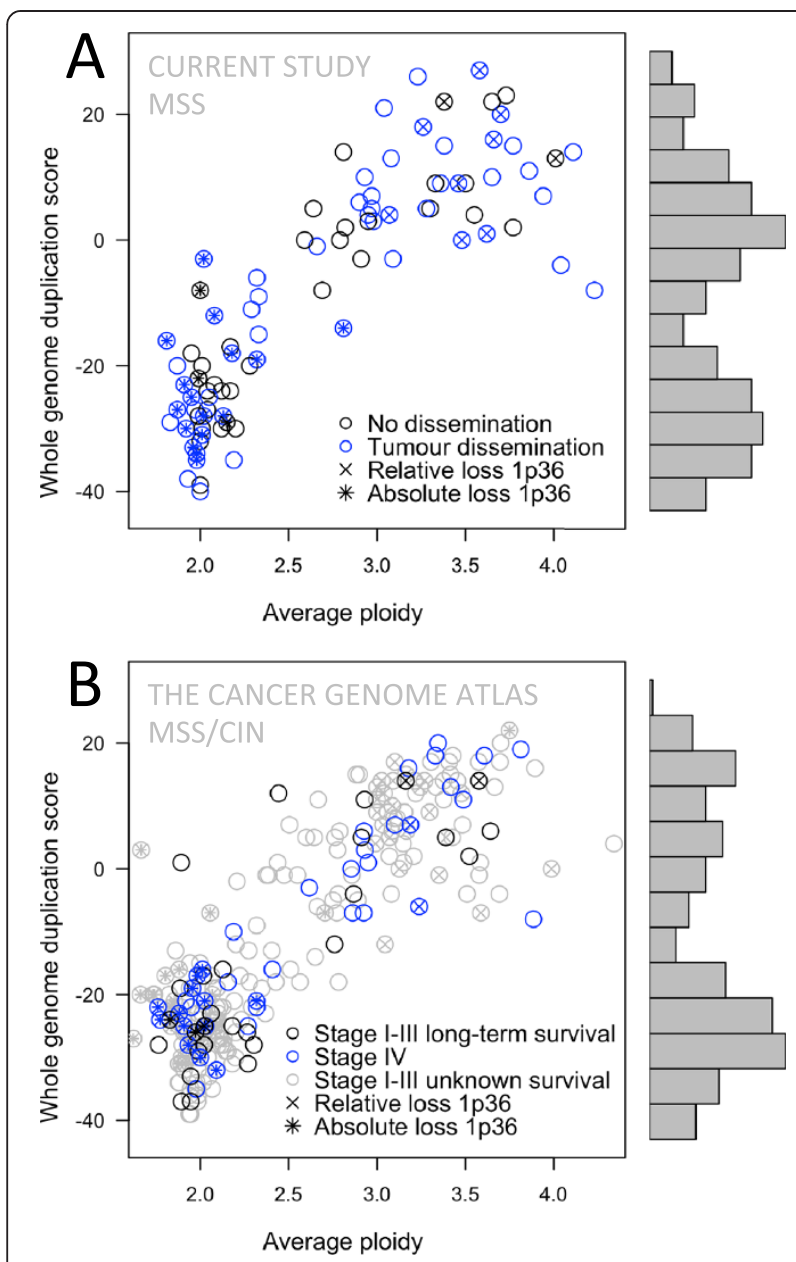

Figure 3 Whole genome duplication. Scatter plots of whole genome duplication (WGD) score and average ploidy indicate strong correlation between hyperploidy and evidence of genome duplication, neither of which associated with tumour dissemination or relative loss of 1 p36. Absolute loss of 1p36 (which is also counted as relative loss) was almost exclusive to near-diploid genomes. The histograms show bi-modal distributions for the WGD score, which suggests two groups of samples; one having undergone WGD and the other not. A) Samples of the current study (MSS, 92) with tumour dissemination (metastatic disease) in blue. B) TCGA validation samples (MSS/CIN, 252), metastatic at diagnosis in blue and with no metastasis at diagnosis and long-term survival in black.

Association between 1p36 loss and metastasis in colon cancer has been described previously [1,18-20]. In this study we have shown that loss on 1p36 is associated with tumour dissemination in MSS tumours of stages II-IV. Stage IV was included in the study as cases with tumour dissemination in order to improve the total number of samples and the estimate of effect size, but significance was retained in the stage II-III subset. Statistical significance was also retained for stage II-III when patients who received adjuvant chemotherapy were excluded, and odds ratios were similar in stage II and stage III separately, supporting that 1 p36 loss can be used as a prognostic marker at diagnosis. While it is not unlikely that this marker can be applied also to MSI cases (disseminated MSI cases were indeed enriched for CNAs similar to those seen in MSS, Additional file 1: Figure S1), the current study included too few MSI cases with dissemination to explore this further.

1p36.11-12 was the most commonly deleted region of 1 p36 in both the current study and in the TCGA validation set. The strongest association with tumour dissemination was seen in a $15 \mathrm{Mbp}$ region of 1p36.11-21 (Figure 2A), similar to the region identified by Thorstensen et al [19]. The region contains multiple genes with a known or suspected role in cancer including ARID1A [21], E2F2 [22], NBPF1 [23], PAX7 [24], RUNX3 [25] and SDHB [26]. One or more such gene may be the cause of worse prognosis through a dosage effect. It should be possible to identify them using a larger number of samples, or using expression analysis of samples without loss of copies, as such a gene may also be down-regulated by other genetic or epigenetic mechanisms.

Assessing the practical value of a marker requires estimating the associated relative and absolute risk. In a selected case-control study such as this one only odds ratio is relevant, as the absolute frequency of distant recurrence and related markers cannot be estimated without an unselected cohort design.

Analysis of absolute allele-specific copy numbers is uncommon in studies of this kind; copy number gain and loss are normally assigned based on log-ratio only (DNA abundance in the extract, along the reference genome, relative to its own median [11]), disregarding tumour cell content and unknown average ploidy of genomes. Absolute loss of copies results in a relatively low copy number relative to the rest of the genome, but $\mathrm{LOH}$ or relative loss may occur without absolute loss to fewer than two copies. We identified relative loss on 1 p36 as a better marker than absolute loss due to a combination of high odds ratio and high total frequency. Relative loss on 1p36 was not associated with hyperploidy or duplication of the genome, while absolute loss was almost exclusive to near diploid genomes. Though the prognostic values of absolute and relative loss on 1p36 were similar (Table 2), relative loss as a prognostic marker would benefit more patients by applying also to hyperploid cancer genomes. It should be noted that if a gene dosage effect is causing the worse prognosis, the effect on prognosis may depend on the size of the dosage effect (e.g. in a genome with four copies on average, 3 remaining copies of 1p36 may lead to a better prognosis than one remaining copy). A much larger number of samples would be required to describe such an effect in detail.

This study was designed to investigate association between genomic aberrations and tumour dissemination as a categorical variable, at diagnosis or within 5 years of 
observation after surgery, and irrespective of time to recurrence or death. Five years is a sufficiently long time to identify virtually all recurrences in colon cancer patients [27]. Relative loss on 1 p36 may be a particularly useful prognostic marker for stage II patients where it, according to our results, motivates the use of adjuvant chemotherapy and regular observation for signs of relapse.

\section{Conclusions}

In this study we have shown that 1 p36 deletion can be used to predict metastatic recurrence in stage II-III patients. The association with metastatic disease was validated in independent data from The Cancer Genome Atlas. Allele-specific copy number analysis allowed the distinction of 1p36 loss relative to individual genome average ploidy as a better prognostic marker than absolute loss of copies, as relative loss had similar prognostic value and was more frequent. This marker may be used to reduce under-treatment particularly in stage II where about $15 \%$ of patients have distant recurrence after treatment primarily based on surgery.

\section{Ethical approval}

This study was approved by the Regional Ethical Review Board of Uppsala (2007/116) and written consent was obtained from participants.

\section{Additional file}

Additional file 1: This file includes supplementary figures, genome-wide copy number estimates and statistics.

\section{Abbreviations \\ CIN: Chromosome Instability; CNA: Copy Number Alteration; LOH: Loss-of-heterozygosity; MSI: Microsatellite Instability; MSS: Microsatellite Stable; OR: Odds Ratio; TCGA: The Cancer Genome Atlas; WGD: Whole Genome Duplication. \\ Competing interests \\ $L M$ and $T S$ are shareholders in ExScale Biospecimen Solutions $A B$, which commercializes the technology for scalable gDNA extraction used.}

\section{Authors' contributions}

Study conception: BG, LP and Al. Collection and analysis of clinical data: HB, MS. Characterization of fresh-frozen biobank material: JB and PM. Molecular analysis: MS, LM, KE and TS. Bioinformatic analysis: MM, HGK and BV. Interpretation of results: MM, HGK and Al. Drafted the manuscript: MM, $\mathrm{Al}$ and HGK. All authors read and approved the final version of the manuscript.

\section{Acknowledgements}

We thank Maria Rydåker, Malin Olsson, Anna Haukkala and Simin Tahmasebpoor for expert technical assistance and acknowledge the support from the Array and Analysis Facility, Science for Life Laboratory, Husargatan 3, 75123 Uppsala, Sweden. Funding for sample preparation and gene analyses was provided by VINNOVA and the Swedish Cancer Society. The results published here are in part based upon data generated by The Cancer Genome Atlas pilot project established by the $\mathrm{NCl}$ and NHGRI. Information about TCGA and the investigators and institutions who constitute the TCGA research network can be found at "http://cancergenome.nih.gov".

\section{Author details}

${ }^{1}$ Science for Life Laboratory, Department of Medical Sciences, Uppsala University, Box 3056, Uppsala 750 03, Sweden. ${ }^{2}$ Department of Surgical Sciences, Uppsala University, Uppsala, Sweden. ${ }^{3}$ Science for Life Laboratory, Department of Immunology Genetics and Pathology, Uppsala University, Uppsala, Sweden. ${ }^{4}$ Department of Radiology, Oncology and Radiation Science, Uppsala University, Uppsala, Sweden. ${ }^{5}$ Leibniz Research Centre for Working Environment and Human Factors, Dortmund TU, Dortmund, Germany.

Received: 29 July 2014 Accepted: 13 November 2014

Published: 24 November 2014

\section{References}

1. Network TCGA: Comprehensive molecular characterization of human colon and rectal cancer. Nature 2012, 487:330-337.

2. Perucho M: Correspondence re: C. R. Boland et al. A National Cancer Institute Workshop on Microsatellite Instability for Cancer Detection and Familial Predisposition: Development of International Criteria for the Determination of Microsatellite Instability in Colorectal Cancer. Cancer Res., 58: 5248-5257, 1998. Cancer Res 1999, 59:249-256.

3. Laiho $P$, Launonen $V$, Lahermo $P$, Esteller M, Guo M, Herman JG, Mecklin J-P, Järvinen $H$, Sistonen $P$, Kim K-M, Shibata D, Houlston RS, Aaltonen LA: Low-level microsatellite instability in most colorectal carcinomas. Cancer Res 2002, 62:1166-1170.

4. Lothe RA, Peltomäki P, Meling Gl, Aaltonen LA, Nyström-Lahti M, Pylkkänen L, Heimdal K, Andersen TI, Møller P, Rognum TO, Fosså SD, Haldorsen T, Langmark F, Brøgger A, de la Chapelle A, Børresen A-L: Genomic instability in colorectal cancer: relationship to clinicopathological variables and family history. Cancer Res 1993, 53:5849-5852.

5. Halling KC, French AJ, McDonnell SK, Burgart LJ, Schaid DJ, Peterson BJ, Moon-Tasson L, Mahoney MR, Sargent DJ, O'Connell MJ, Witzig TE, Farr GH Jr, Goldberg RM, Thibodeau SN: Microsatellite instability and 8p allelic imbalance in stage B2 and C colorectal cancers. J Natl Cancer Inst 1999, 91:1295-1303.

6. Fearon ER, Cho KR, Nigro JM, Kern SE, Simons JW, Ruppert JM, Hamilton SR, Preisinger AC, Thomas G, Kinzler KW: Identification of a chromosome 18q gene that is altered in colorectal cancers. Science 1990, 247:49-56.

7. Tanaka T, Watanabe T, Kazama Y, Tanaka J, Kanazawa T, Kazama S, Nagawa H: Chromosome $18 \mathrm{q}$ deletion and Smad4 protein inactivation correlate with liver metastasis: a study matched for $\mathrm{T}$ - and $\mathrm{N}$ - classification. $\mathrm{Br} J$ Cancer 2006, 95:1562-1567.

8. Sheffer M, Bacolod MD, Zuk O, Giardina SF, Pincas H, Barany F, Paty PB, Gerald WL, Notterman DA, Domany E: Association of survival and disease progression with chromosomal instability: a genomic exploration of colorectal cancer. Proc Natl Acad Sci U S A 2009, 106:7131-7136.

9. Nakao M, Kawauchi S, Uchiyama T, Adachi J, Ito H, Chochi Y, Furuya T, Oga A, Sasaki K: DNA copy number aberrations associated with the clinicopathological features of colorectal cancers: Identification of genomic biomarkers by array-based comparative genomic hybridization. Oncol Rep 2011, 25:1603-1611.

10. Poulogiannis G, Ichimura K, Hamoudi RA, Luo F, Leung SY, Yuen ST, Harrison DJ, Wyllie AH, Arends MJ: Prognostic relevance of DNA copy number changes in colorectal cancer. J Pathol 2010, 220:338-347.

11. Rasmussen $M$, Sundstrom M, Goransson Kultima $H$, Botling J, Micke P, Birgisson H, Glimelius B, Isaksson A: Allele-specific copy number analysis of tumor samples with aneuploidy and tumor heterogeneity. Genome Biol 2011, 12:R108

12. Carter SL, Cibulskis K, Helman E, McKenna A, Shen H, Zack T, Laird PW, Onofrio RC, Winckler W, Weir BA, Beroukhim R, Pellman D, Levine DA, Lander ES, Meyerson M, Getz G: Absolute quantification of somatic DNA alterations in human cancer. Nat Biotechnol 2012, 30:413-421.

13. Mathot $L$, Wallin M, Sjöblom T: Automated serial extraction of DNA and RNA from biobanked tissue specimens. BMC Biotechnol 2013, 13:66.

14. Kim T-M, Laird PW, Park PJ: The landscape of microsatellite instability in colorectal and endometrial cancer genomes. Cell 2013, 155:858-868.

15. Olshen $A B$, Venkatraman ES, Lucito R, Wigler M: Circular binary segmentation for the analysis of array-based DNA copy number data. Biostatistics 2004, 5:557-572.

16. Fodde R, Kuipers J, Rosenberg C, Smits R, Kielman M, Gaspar C, van Es JH, Breukel C, Wiegant J, Giles RH, Clevers H: Mutations in the APC tumour 
suppressor gene cause chromosomal instability. Nat Cell Biol 2001 3:433-438.

17. Barber TD, McManus K, Yuen KWY, Reis M, Parmigiani G, Shen D, Barrett I, Nouhi Y, Spencer F, Markowitz S, Velculescu VE, Kinzler KW, Vogelstein B, Lengauer C, Hieter P: Chromatid cohesion defects may underlie chromosome instability in human colorectal cancers. Proc Natl Acad Sci U S A 2008, 105:3443-3448.

18. Ogunbiyi OA, Goodfellow PJ, Gagliardi G, Swanson PE, Birnbaum EH, Fleshman JW, Kodner IJ, Moley JF: Prognostic value of chromosome 1p allelic loss in colon cancer. Gastroenterology 1997, 113:761-766.

19. Thorstensen L, Qvist H, Heim S, Liefers GJ, Nesland JM, Giercksky KE, Lothe RA: Evaluation of $1 p$ losses in primary carcinomas, local recurrences and peripheral metastases from colorectal cancer patients. Neoplasia 2000, 2:514-522.

20. Kim M-Y, Yim S-H, Kwon M-S, Kim T-M, Shin S-H, Kang H-M, Lee C, Chung Y-J: Recurrent genomic alterations with impact on survival in colorectal cancer identified by genome-wide array comparative genomic hybridization. Gastroenterology 2006, 131:1913-1924.

21. Wang K, Kan J, Yuen ST, Shi ST, Chu KM, Law S, Chan TL, Kan Z, Chan ASY, Tsui WY, Lee SP, Ho SL, Chan AKW, Cheng GHW, Roberts PC, Rejto PA, Gibson NW, Pocalyko DJ, Mao M, Xu J, Leung SY: Exome sequencing identifies frequent mutation of ARID1A in molecular subtypes of gastric cancer. Nat Genet 2011, 43:1219-1223.

22. Lee C, Chang JH, Lee HS, Cho Y: Structural basis for the recognition of the E2F transactivation domain by the retinoblastoma tumor suppressor. Genes Dev 2002, 16:3199-3212.

23. Vandepoele K, Van Roy N, Staes K, Speleman F, van Roy F: A novel gene family NBPF: intricate structure generated by gene duplications during primate evolution. Mol Biol Evol 2005, 22:2265-2274.

24. Blake J, Ziman MR: Aberrant PAX3 and PAX7 expression. A link to the metastatic potential of embryonal rhabdomyosarcoma and cutaneous malignant melanoma? Histol Histopathol 2003, 18:529-539.

25. Soong R, Shah N, Peh BK, Chong PY, Ng SS, Zeps N, Joseph D, Salto-Tellez M, lacopetta B, Ito Y: The expression of RUNX3 in colorectal cancer is associated with disease stage and patient outcome. Br J Cancer 2009, 100:676-679.

26. Neumann HPH, Pawlu C, Peczkowska M, Bausch B, McWhinney SR, Muresan M, Buchta M, Franke G, Klisch J, Bley TA, Hoegerle S, Boedeker CC, Opocher G, Schipper J, Januszewicz A, Eng C, European-American Paraganglioma Study Group: Distinct clinical features of paraganglioma syndromes associated with SDHB and SDHD gene mutations. JAMA 2004, 292:943-951.

27. Birgisson $\mathrm{H}$, Wallin $\mathrm{U}$, Holmberg L, Glimelius B: Survival endpoints in colorectal cancer and the effect of second primary other cancer on disease free survival. BMC Cancer 2011, 11:438.

doi:10.1186/1471-2407-14-872

Cite this article as: Mayrhofer et al: 1 p36 deletion is a marker for tumour dissemination in microsatellite stable stage II-III colon cancer. BMC Cancer 2014 14:872.

\section{Submit your next manuscript to BioMed Central and take full advantage of:}

- Convenient online submission

- Thorough peer review

- No space constraints or color figure charges

- Immediate publication on acceptance

- Inclusion in PubMed, CAS, Scopus and Google Scholar

- Research which is freely available for redistribution 